American Medical Journal 2 (2): 98-103, 2011

ISSN 1949-0070

(C) 2011 Science Publications

\title{
Role of Cardioprotective Diet in Acute Coronary Syndrome
}

\author{
${ }^{1}$ R.B. Singh, ${ }^{2}$ Suniti Dharwadkar, ${ }^{3}$ Fabien DeMeester, \\ ${ }^{4}$ Lekh Juneja and ${ }^{5}$ Daniel Pella \\ ${ }^{1}$ Department of Internal Medicine, Halberg Hospital and Research Institute, \\ Moradabad, 10(UP) 244001, India \\ ${ }^{2}$ Department of Biochemistry, Saraswati Bhuvan College of Science, \\ Aurangabad, 431001(MS), India \\ ${ }^{3}$ Department of Food and Nutrition, \\ Columbus Paradigm Institute, Bastogne, Belgium \\ ${ }^{4}$ Department of Food and Nutrition, Taiyo Kagaku, Yokkaichi, Japan \\ ${ }^{5}$ Department of Food and Nutrition, Faculty of Medicine, \\ Safaric University, Kosice, Slovakia
}

\begin{abstract}
Problem statement: Nutritional factors appear to be quite important in the pathogenesis and prognosis among patients of Acute Myocardial Infarction (AMI) or Acute Coronary Syndrome (ACS). Increased consumption of proinflammatory foods; such as refined starches and sugar, trans fat, saturated fat and w-6 fatty acids may increase proinflammatory cytokines, oxidative stress and free fatty acids causing endothelial dysfunction. Approach: Columbus foods with low w-6/w-3 ratio of fatty acids, may play a protective role. Many adverse parameters such as higher concentrations of proinflammatory cytokines, transcription factors and adhesion molecules, free radicals and decrease in nitrite levels were shown to be related to the dietary composition and thus may serve as independent predictors of ACS. Diets rich in w-3 fatty acids, vitamins, minerals and antioxidants and low in refined carbohydrates cause decrease in levels of plasma lipid peroxides, antioxidant vitamins, serum Glutamic Oxaloacetate Transaminase (SGOT) and Lactate Dehydrogenase (LDH) cardiac enzymes in patients with ACS. Results: Available evidence indicate that cardioprotective diet can decrease sudden cardiac deaths, total cardiac deaths, non-fatal infractions and total cardiac events among patients receiving such diet compared to standard diet. The existing evidence support that diet rich in w- 3 fatty acids, vitamins, minerals and antioxidants with low refined carbohydrates may be beneficial to vascular endothelium and myocardium and thus seems to function as cardioprotective in patients with ACS. Conclusion: The Western foods that lack essential vitamins, minerals and w-3 fatty acids and that are rapidly absorbed should be avoided in patients with ACS. More studies especially in the context of developing countries are required for establishing the vital role of the cardio protective diet.
\end{abstract}

Key words: Acute Myocardial Infarction (AMI), Acute Coronary Syndrome (ACS), proinflammatory cytokines, transcription factors, adhesion molecules, endothelial dysfunction, dietary intervention, cardioprotective diet, fatty acids ratio, antidepressants, antioxidants

\section{INTRODUCTION}

The Acute Coronary Syndromes (ACS) encompass a spectrum of unstable coronary artery disease from unstable angina to Acute Myocardial Infarction (AMI) .It has been reported that the risk of death in acute myocardial infarction AMI or ACS before admission to hospital was up to $20 \%$. However, the death rates after admission to the hospital were decreased significantly; upto $7 \%$ patients die before discharge and the risk of death continued to be high for six months after the AMI.Apart from the risk of mortality, a similar risk remains for reinfarction after admission to the hospital in the next one year after discharge but would depend upon modification of risk factors and mode of treatment (Liew et al., 2006; Teo et al., 2007; Singh et al., 1992; 2001; 2004; 2006). Further, though the risk predictors of nonfatal AMI (NFAMI) have been studied (www.Columbus- concept.com), the risk factors in patients with NFAMI ignore risk among those who died

Corresponding Author: R.B. Singh, Department of Internal Medicine, Halberg Hospital and Research Institute, Moradabad, 10(UP) 244001, India 
Am. Med.J. 2 (2): 98-103, 2011

due to greater risk before coming to the hospital and many patients with triggers of AMI may die because of sudden cardiac death. Although in this aspect, the correct levels of risk factors among population of Costa Rica were not represented this study (www.Columbusconcept.com), the study signified a noteworthy attempt to indicate to the western investigators that the levels of risk factors in the developing world may be different than that of developed countries. In this context, diet and lifestyle guidelines for prevention computed for the developed countries have to be modified for the developing world.

Genetic and nutritional factors and mal adaptations appear to be quite important in the pathogenesis and prognosis among patients with AMI (Liew et al., 2006; Teo et al., 2007; Singh et al., 1992). Jordanian patients with AMI have been shown to have increased plasma lipoprotein (a) levels. AMI may be due to thrombosis in the unstable plaque and may be associated with activation of neurohormones and proinflammatory cytokines, resulting into neuroendocrine dysfunction, which worsens the prognosis in AMI. There may be periodontal disease, oxidative stress, hyperglycemia, hyperinsulinemia and hyper triglyceridemia, increased levels of catecholamines, free fatty acids and cortisol. Increased consumption of proinflammatory foods, such as refined starches and sugar, trans fatty acids, w- 6 fatty acids and saturated fat and Trans Fatty Acids (TFA) may increase free fatty acids, oxidative stress and proinflammatory cytokines, causing endothelial dysfunction (Singh et al., 2001; 2006; 2004). Therefore consuming proinflammatory foods could be an important predictor and cause of bad prognosis in ACS, because these patients have a preexisting proinflammatory milieu in association with atherothrombosis (Singh et al., 1992; 2001; 2004; 2006). In this regard, Columbus foods with proper w6/w-3 ratio of fatty acids may play a protective role. Hypercholesterolemia is known risk factor for heart disease such as atherosclerosis and AMI and certain dietary supplements have been shown to possess hypolipedemic effects and thus may be useful in the treatment.

In one study, among 54 patients with Acute Coronary Syndromes (ACS), 41 patients had Acute Myocardial Infarction (AMI), 5 possible MI, 4 cases unstable angina and rest 4 angina pectoris (Singh et al., 2004). The control subjects $(n=85)$ were randomly selected from the population, drawn from a similar age and sex of the subjects. Increased consumption of TFA ( $>5 \mathrm{~g} /$ day) was significantly greater among ACS patients compared to controls (50.0 Vs. 0.0\%,). Large meals (>1000 K cal) (50.0 Vs. $29.4 \%$,) and large breakfast (40.7-11.8\%), rich in TFA, linoleic acid, saturated fat and refined carbohydrates intakes were also significantly greater among ACS patients, compared to control subjects.

Serum level of nitrite (an indicator of Nitric Oxide, NO) was significantly decreased in patients with ACS compared to controls (mean + S.D. mean + SD:0.36+ 1.42 Vs. $0.96+1.48 \mathrm{uM}$, Confidence Interval (CI) difference 0.60,0.34-1.02). After 4 weeks of follow up, serum nitrite level recovered showing significant increase without such changes in the control group $(0.88$ Vs. $1.09 \mathrm{uM}$; CI $0.21,0.67-1.12)$. The incidence of lipoprotein(a) excess ( $\left.>30 \mathrm{mg} \mathrm{dL}^{-1}\right)$ and mean levels of lipoprotein(a) (difference $6.4 \mathrm{mg} \mathrm{dL}^{-1}, 95 \%$ confidence interval 2.8-10.5) was significantly greater in the ACS group compared to control subjects. Serum levels of vitamin $\mathrm{E}$, vitamin $\mathrm{C}$, beta-carotene, coenzyme Q10, magnesium and potassium were declined significantly and insulin, glucose, triglycerides and lipoprotein (a), Tumor Necrosis Factor (TNF)-alpha and Interleukin 6 (IL-6) were significantly raised in ACS compared to their levels after 4 weeks of follow up.

There was a significantly greater occurrence of acute ACS events in the second quarter of the day (40.7 vs. 18.3, 12.9, 27. 7\%), compared to other quarters. A circadian rhythm was also noted for serum level of NO showing significantly lower nitrite in 6.00-12.00 AM period compared to afternoon, $12.00-18.00 \mathrm{~h}$ period $(0.27+1.12$ Vs. $0.42+1.44 \mathrm{uM})$. The differences in the nitrite levels in the two groups indicated that low levels of nitrite appear to be a risk factor of ACS. The lower levels of nitrite during acute stage of AMI and its recovery after 4 weeks of follow up implied that transient hyperglycemia and hyperinsulinemia may also be responsible for its increase, (apart from low arginine intake and sedentary behavior) by inhibiting the expression of $\mathrm{NO}$ receptors caused by metabolic reactions evoked by ACS. Reduction in antioxidants vitamins may be due to increased oxidative stress which is common in ACS due to enhanced free radical generation which may also influence NO levels and cause endothelial dysfunction. The risk factors related to lipid profile in cardiovascular diseases and AMI have been shown to be associated with the elevated indices of oxidative stress with concomitant decrease in serum antioxidant vitamins levels.

In India AMI, diabetes and hypertension are more common among higher social classes compared to lower social classes, possibly due to lower fat intake and enormous occupational physical activity in lower social classes (Singh et al., 2005). Though the roles of various risk factors and dietary fatty acids, tobacco, 
physical inactivity, abdominal obesity and alcohol intake, as risk factors of AMI have been studied (Kabagambe et al., 2007), there is no mention of the role of refined starches and sugar, large meals, decreased intake of fruits, vegetables and nuts and serum nitrite levels as risk predictors of AMI. A circadian rhythm of AMI in the second quarter of $24 \mathrm{~h}$ was also reported (Singh et al., 2004). There was increased platelet aggregation and blood pressure variability and decreased heart rate variability in association with higher concentrations of catecholamines, oxidative stress and cytokines between 6.00-12.00 $\mathrm{h}$ in the morning .Eating a heavy breakfast rich in proinflammatory foods; TFA, linolenic acid and refined starches and low in w-3 fatty acids may have predisposed the patients to AMI in various studies (Singh et al., 2001; 2004). There was an increased superoxide ion generation in leukocytes and mononuclear cells, on glucose ingestion in normal subjects, with enhanced activity of nuclear factor- $\mathrm{\kappa B}(\mathrm{NF}-\mathrm{\kappa B})$, a transcriptional factor regulating the activity of at least 125 genes, most of which are pro inflammatory (Singh et al., 2006). Refined carbohydrates also caused an increase in two other pro-inflammatory transcription factors, Activating Protein-1(AP-1) and Early Growth response protein-1 (Egr-1), the first regulating the transcription of matrix metallo-proteinases and the second modulating the transcription of tissue factor and plasminogen activator inhibitor-1.These adverse factors related to diet may be independent predictors of ACS.

The consumption of a mixed meal resulted in an activation of (NF- $\mathrm{kB}$ ) which is known to be associated with the generation of Reactive Oxygen Species (ROS) by mononuclear cells. Superoxide anion appears to be an activator of at least two major pro-inflammatory transcription factors, NF- $\mathrm{\kappa B}$ and AP-1. Previous findings, demonstrated that after oral or intravenous glucose challenges, in both normal subjects and in the patients with type 2 diabetes mellitus, there was an increased generation of ROS and raised circulating levels of proinflammatory cytokines, such as TNF- $\alpha$, IL-6 and Interleukin 18 (IL-18) (Singh et al., 2006). A single high-fat meal produced endothelial activation, as evidenced by increased concentrations of the adhesion molecules Vascular Cell Adhesion Molecule-1 (VCAM-1) and Intercellular Adhesion Molecule-1 (ICAM-1), in association with raised plasma concentrations of IL-6 and TNF- $\alpha$. (Singh et al., 1992; 2001; 2004; 2006). A high-fat meal which may be large, may increase the circulating levels of IL-18, a pro-inflammatory cytokine supposed to be involved in plaque destabilization associated with the simultaneous decrease of circulating adiponectin, an adipocytederived protein with insulin sensitizing, anti- inflammatory and antiatherogenic properties. However, eating a high-fat meal together with vegetable foods rich in natural antioxidants largely prevented the negative effects on endothelial function. Endothelial dysfunction acutely triggered by the consumption of a high-fat meal rich in saturated fatty acids was reduced by the simultaneous consumption of a vegetable serving including pepper $(100 \mathrm{~g})$ tomatoes $(100 \mathrm{~g})$ and carrots $(200 \mathrm{~g})$. It seems that these foods are slowly digested and absorbed without causing any significant increase in free radical stress and free fatty acids, which is a characteristic of Columbus foods and therefore such foods may improve the prognosis in AMI (Singh et al., 2006; 2004).

The proinflammatory cytokines are known to insult the neurons which worsen in the deficiency of w-3 fatty acids which are responsible for the survival of the neurons. It has been suggested that the $\mathrm{w}-3$ fatty acids can regulate leptin gene expression and the concentrations of anandamides in the brain, which in turn binds to endogenous cannabinoid receptors and regulate food intake and satiety. In the Indian experiments (Cornelissen et al., 2007; Singh et al., 1992; 1995; 2001; 2007; 2010), a diet rich in w-3 fatty acids ( 1.8 Vs $0.5 \mathrm{~g}$ day $^{1}$ alpha linolenic acid) showed significant benefit compared to the control groups. (12).Clinical trials with anti-inflammatory foods, coenzyme Q10, w-3 fatty acids and statins in patients with proven proinflammatory status with due consideration to diet will be helpful as their pleiotropic effects may prove beneficial in this setting (De Lorgeril et al., 1999; De Meester, 2011).

Clinical trials, using the Mediterranean food approach are also in line with the above experimental and epidemiological evidence. In the Lyon Diet Heart Study (De Lorgeril et al., 1994; 1999; Renaud et al., 1995), 605 patients who had a myocardial infarction were randomly assigned to a 'Mediterranean-style' diet or a control diet resembling the American Heart Association Step I diet. The Mediterranean diet model supplied $30 \%$ of energy from fats and $<10 \%$ of energy from saturated fatty acids, whereas the intake of 18:3 (n-3) ( $\alpha$-linolenic acid) provided $>0.6 \%$ of energy. After a mean follow-up of 27 months, the risk of new acute myocardial infarction and episodes of unstable angina was reduced by $\sim 70 \%$ by the Mediterranean diet; moreover, total mortality was also reduced by $70 \%$.

The role of an 'Indo-Mediterranean diet' in 1000 patients in India, with existing coronary disease or at high risk for coronary disease was studied by Singh et al. (2002). When compared with the control diet, the intervention diet was characterized by increased intake of mustard or soybean oil, nuts (walnuts, almonds), vegetables, fruits and whole grains. 
Am. Med. J. 2 (2): 98-103, 2011

Table 1: Comparison of dietary intake of intervention group and control group of acute myocardial infarction after one year intake ( (tubers not included)

\begin{tabular}{|c|c|c|}
\hline & Intervention group $(\mathrm{n}=204)$ & Control group $(n=202)$ \\
\hline \multicolumn{3}{|l|}{ Foods and nutrients } \\
\hline Fruits and vegetables $\left(\mathrm{g} \mathrm{day}^{-1}\right)$ & $575 \pm 91.4 * *$ & $220.5 \pm 19.6$ \\
\hline Legumes and pulses $\left(\mathrm{g} \mathrm{day}^{-1}\right)$ & $95.0 \pm 8.9^{* *}$ & $45.6 \pm 5.60$ \\
\hline Almonds and walnuts $\left(\mathrm{g} \mathrm{day}^{-1}\right)$ & $75.5 \pm 5.2 * *$ & - \\
\hline Fish $\left(\right.$ g day $\left.^{-1}\right)$ & $22.4 \pm 4.1 * *$ & $10.5 \pm 3.50$ \\
\hline Chicken $\left(\mathrm{g} \mathrm{day}^{-1}\right)$ & $10.2 \pm 3.2 *$ & $66.5 \pm 10.5$ \\
\hline Mustard or soybean oil & $31.5 \pm 5.5 * *$ & $6.8 \pm 2.80$ \\
\hline Butter or clarified butter (g. day $\left.{ }^{-1}\right)$ & $3.3 \pm 0.71 *$ & $12.6 \pm 3.50$ \\
\hline Skim milk $\left(\mathrm{mL} \mathrm{day}^{-1}\right)$ & $152 \pm 14.5^{*}$ & $165.5 \pm 16.1$ \\
\hline Wheat chapatti & $30.6 \pm 5.5$ & $55.6 \pm 7.80$ \\
\hline Bread, biscuits $\left(\mathrm{g} \mathrm{day}^{-1}\right)$ & $25.5 \pm 6.2 * *$ & $212.2 \pm 18.1$ \\
\hline Rice and wheat cereals $\left(\mathrm{g} \mathrm{day}^{-1}\right)$ & $30.6 \pm 5.5$ & $35.6 \pm 4.80$ \\
\hline Honey or raisins $\left(\mathrm{g} \mathrm{day}^{-1}\right)$ & $5.5 \pm 1.2$ & - \\
\hline Sugar $\left(\mathrm{g} \mathrm{day}^{-1}\right)$ & $12.6 \pm 3.4 *$ & $30.5 \pm 7.600$ \\
\hline Alpha-linolenic acid $\left(\mathrm{g} \mathrm{day}^{-1}\right)$ & $1.8 \pm 0.66^{* *}$ & $0.65 \pm 0.14$ \\
\hline Ascorbic acid $\left(\mathrm{mg} \mathrm{day}^{-1}\right)$ & $440.6 \pm 100.6^{* *}$ & $160 \pm 38.0000$ \\
\hline
\end{tabular}

Table 2: Effect of Cardioprotective diet on cardiac events (after one year )

\begin{tabular}{lll}
\hline & $\begin{array}{l}\text { Intervention } \\
\text { group }(\mathrm{n}=204)\end{array}$ & $\begin{array}{l}\text { Control } \\
\text { group }(\mathrm{n}=202)\end{array}$ \\
\hline Non-fatal myocardial infarction & $30(15)^{* *}$ & $48(24)$ \\
Fatal myocardial infarction & $13(6)^{*}$ & $19(9)$ \\
Sudden cardiac death $(<1 \mathrm{~h})$ & $7(3)^{*}$ & $15(7)$ \\
Suspected cardiac death & - & $1(0.5)$ \\
Total cardiac mortality & $20(10)^{* *}$ & $34(17)$ \\
Total cardiac events and deaths & $51(25)^{* *}$ & $86(43)$ \\
\hline
\end{tabular}

$\mathrm{P}$ value was obtained by comparison of intervention and control group by a $\mathrm{Z}$ test for proportions. $*:=\mathrm{p}<0.05, * *:=\mathrm{p}<0.01$. Values in parenthesis are number (\%) Modified from (Singh et al., 1997; 1992)

There was a significant reduction in the rate of fatal myocardial infarction by $33.3 \%$ and the rate of sudden death from cardiac causes by $66.6 \%$ in the intervention group.

Moreover, the vitamins and minerals can prevent stress and depression and the Mediterranean diet rich in these can protect depression and thus may be beneficial for heart problems as well. Recently banana peels have been shown to have antidepressant activity which may have implications in the treatment of AMI. Banana contains serotonin and serotonin deficiency is a risk factor for depression which in turn been related both to the development of heart disease and to prognosis in patients following AMI. Nutritional deficiency of essential vitamins, minerals and $\mathrm{w}-3$ fatty acids can be correlated with certain mental disorders. We propose that induced systems including dietary pattern along with the thoughts and experience can change the configuration of our brain connections and may trigger AMI because acute stress and elevated depressive symptoms appear to be risk factors and prognostic marker of cardiovascular disease. It is possible that the
Western foods that are rapidly absorbed should be avoided and the diet rich in the vitamins and antioxidants, known to prevent mind-body interactions and beneficial to vascular endothelium and myocardium is desirable in patients with ACS.

There is limited evidence regarding the role of dietary intervention in patients with AMI (De Lorgeril et al., 1999; De Lorgeril et al., 1994; Renaud et al., 1995; Singh et al., 2002). The effects of a diet rich in w-3 fatty acids, vitamins, minerals and antioxidants (fruits, vegetables, legumes, walnuts, almonds, fish, mustered oils)and low in refined carbohydrates, in patients with AMI were studied (Table 1) (Singh et al., 1992; 1997). All patients with a diagnosis of ACS were assigned to an intervention diet $(n=204)$ or a control $\operatorname{diet}(\mathrm{n}=202)$ within $48 \mathrm{hrs}$ of the onset of the symptoms of AMI. The intervention group was advised to consume $600 \mathrm{~g} \mathrm{day}^{-1}$ of fruits, vegetables, legumes and almonds and walnuts, in a soup or semisolid form. Tomato soup, skim milk and curd (yogurt) were commonly used to mix crushed almonds and walnuts and other foods, which were grilled with mustered oil. The control group was advised a low fat diet consistent with National Cholesterol Education Program. (Table 1). Clinical characteristics, time elapsed from symptom onset to the index infarction, site of infarction; drug therapy and final diagnosis were comparable between the two groups. Intake of foods and selected nutrients was assessed during the 1 week and after 1 year. After 1 week, plasma lipid peroxides, vitamin $\mathrm{C}$ and lactate dehydrogenase levels were determined (Fig. 1). A modest improvement in dyslipidemia and a decrease in oxidative damage were observed in the fish oil and mustard oil groups but not in the placebo group (Singh et al., 1995). 
Am. Med.J. 2 (2): 98-103, 2011

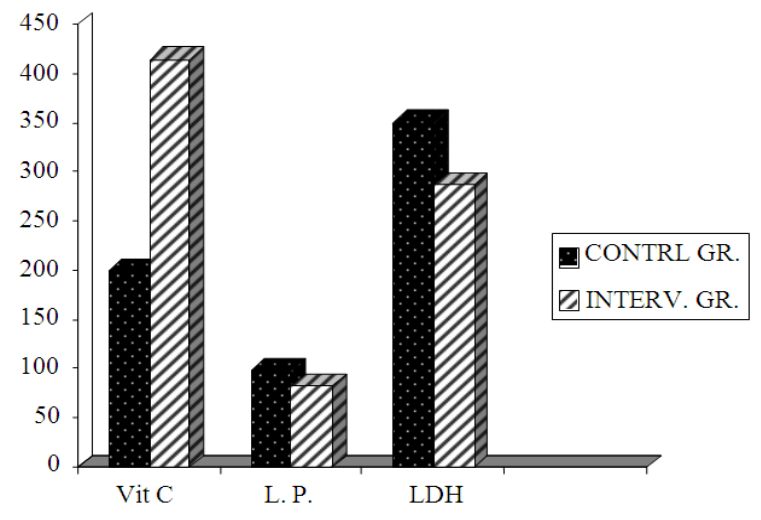

Fig.1: Effect of dietary alpha-linolenic acid intervention (after one week) percentage difference in the serum Vitamin C (Vit C), Lipid Peroxides (LP) and Lactate Dehydrogenase (LDH) Modified from (Singh et al., 1995)

Compared with the control groups, sudden cardiac deaths, total cardiac deaths, non-fatal infarctions and total cardiac events were also significantly reduced in the intervention group (Table 2) (Singh et al., 1997). Dietary w-3 fatty acids have been shown to reduce mortality post-MI. Recent evidence also support the consumption of polyunsaturated fats especially w-3 fatty acids, instead of saturated fats as a measure of decreasing coronary heart disease (Mozaffarian et al., 2010).

\section{CONCLUSION}

In conclusion, many adverse parameters such as proinflammatory cytokines (TNF- $\alpha$, , IL-6 and IL-18) and transcriptional factors (NF- $\mathrm{kB}, \mathrm{AP}-1$ and Erg-1), adhesion molecules (VCAM-1 and ICAM-1), generation of free radicals and oxidative stress and declined in NO levels and antioxidant vitamins related to the dietary composition may serve as independent predictors of ACS. The existing evidence indicates that diet rich in w-3 fatty acids, vitamins, minerals and antioxidants with low refined carbohydrates are beneficial to vascular endothelium and myocardium and thus found to be cardioprotective in patients with ACS. The Western foods that lack essential vitamins, minerals and w-3 fatty acids and that are rapidly absorbed should be avoided in patients with ACS. More studies especially in the context of developing countries will shed light on the imperative role of the cardioprotective diet.

\section{REFERENCES}

Cornelissen, G., F. Halberg, K. Otsuka, R.B. Singh and C.H. Chen, 2007. Chronobiology predicts actual and proxy outcomes when dipping fails. Hypertension, 49: 237-239. DOI: 10.1161/01.HYP.0000250392.51418.64 PMID: 17075031

De Lorgeril, M., P. Salen, J.L. Martin, I. Monjaud and J. Delaye et al., 1999. Mediterranean diet, traditional risk factors, and the rate of cardiovascular complications after myocardial infarction: Final report of the Lyon Diet Heart Study. Circulation, 99: 779-785. PMID: 9989963

De Lorgeril, M., S. Renaud, N. Mamelle, P. Salen and J.L. Martin et al., 1994. Mediterranean alphalinolenic acid-rich diet in secondary prevention of coronary heart disease. Lancet, 343: 1454-1459. DOI: 10.1016/S0140-6736(94)92580-1

De Meester, F., 2011. Impact of research conducted by Dr Ram B singh on modern concepts of nutrition and lifestyle in mind-body diseases. Open Nutr. J., 4: 31-33. DOI: $10.2174 / 1876396001104010031$

Kabagambe, E.K., A. Baylin and H. Campos, 2007. Nonfatal acute myocardial infarction in Costa Rica. Circulation, 115: 1075-1081. DOI: 10.1161/CIRCULATIONAHA.106.643544 PMID: 17339565

Liew, R., S. Sulfi, R. Ranjadayalan, J. Cooper and A.D. Timmis, 2006. Declining case fatality rates for acute myocardial infarction in South Asian and white patients in the past 15 years. Heart, 92: 10301034. DOI: $10.1136 / \mathrm{hrt} .2005 .078634$ PMID: 16387823

Mozaffarian, D., R. Micha and S. Wallace, 2010. Effects on coronary heart disease of increasing polyunsaturated fat in place of saturated fat: A systematic review and meta-analysis of randomized controlled trials. PLoS Med., 7: e1000252. PMID: 20351774

Renaud, S., M. Lorgeril, J. Delaye, J.J. Guidollet and F. Jacquard et al., 1995. Cretan mediterranean diet for prevention of coronary heart disease. Am. J. Clin. Nutr., 61: 1360S-1367S. PMID: 7754988

Singh, R.B, M.A. Niaz, J.P. Sharma, R. Kumar and V. Rastogi et al., 1997. Randomized, double-blind, placebo-controlled trial of fish oil and mustard oil in patients with suspected acute myocardial infarction: The Indian experiment of infarct survival-4. Cardiovasc. Drug Ther., 11: 485-491. DOI: 10.1023/A:1007757724505 PMID: 9310278 
Singh, R.B., D. Pella and F. DeMeester, 2006. What to 'eat and chew' in acute myocardial infarction? Eur. Heart J., 27: 1628-1629. DOI: 10.1093/eurheartj/ehl035 PMID: 16717073

Singh, R.B., D. Pella, J.P. Sharma, S. Rastogi and K. Kartikey et al., 2004. Increased concentrations of lipoprotein(a), circadian rhythms and metabolic reactions evoked by acute myocardial infarction, associated with acute reactions in relation to large breakfasts. Biomed. Pharmacother., 58: 116-122. DOI: 10.1016/S0753-3322(04)80019-2

Singh, R.B., F. De Meester and A. Wilczynska, 2010. The tsim tsoum approaches for prevention of cardiovascular disease. Cardiol. Res. Prac., 18-18. DOI: 10.4061/2010/824938 PMID: 20671994

Singh, R.B., F. De Meester, F. Juneja, V. Mechirova and D. Pella, 2007. New risk factors of heart failure. Eur. Heart J. DOI: 10.1093/eurheartj/ehm006

Singh, R.B., G. Dubnov, M.A. Niaz, S. Ghosh and R. Singh et al., 2002. Effect of an Indo-Mediterranean diet on progression of coronary artery disease in high risk patients (Indo-Mediterranean Diet Heart Study): A randomised single-blind triall. Lancet, 360: 1455-1461. DOI: 10.1016/S01406736(02)11472-3

Singh, R.B., M.A. Niaz and C. Kartik, 2001. Can n-3 fatty acids provide myocardial protection by decreasing infarct size and inhibiting atherothrombosis? Eur. Heart J., 3: 62-69. DOI: $10.1016 / \mathrm{S} 1520-765 \mathrm{X}(01) 90122-1$
Singh, R.B., M.A. Niaz, P. Agarwal, R. Beegum and S.S. Rastogi, 1995. Effect of antioxidant-rich foods on plasma ascorbic acid, cardiac enzyme, and lipid peroxide levels in patients hospitalized with acute myocardial infarction. J. Am. Diet. Assoc., 95: 775-780. DOI: 10.1016/S0002-8223(95)00215-4

Singh, R.B., S.S. Rastogi, R. Verma, B. Laxmi and R. Singh et al., 1992. Randomised controlled trial of cardioprotective diet in patients with recent acute myocardial infarction: results of one year follow up. BMJ. 304: 1015-1019. DOI: 10.1136/bmj.304.6833.1015 PMID: 1586782

Singh, R.B., S.S. Rastogi, R. Verma, L. Bolaki and R.S. BSc, 1992. An Indian experiment with nutritional modulation in acute myocardial infarction, Am. J. Cardiol., 69: 879-885. DOI: 10.1016/00029149(92)90786-X

Singh, R.B., V. Singh, S.K. Kulshrestha, S.Singh and P. Gupta et al., 2005. Social class and all-cause mortality in an urban population of North India. Acta Cardiol., 60: 611-176. DOI: 10.2143/AC.60.6.2004933 PMID: 16385922

Teo, M., S. Lalondrel, M. Roughton, R.G. Mason and S.W. Dubrey, 2007. Acute coronary syndromes and their presentation in Asian and Caucasian patients in Britain. Heart, 93: 183-188. DOI: 10.1136/hrt.2006.091900 PMID: 16914486 